\title{
Combined preclosure technique and traction method facilitating endoscopic full-thickness resection of a gastric fundal gastrointestinal stromal tumor
}

We describe the case of a 47-year-old man with a gastric fundal mass found on upper gastrointestinal endoscopy ( Fig.1a). Endosonography showed a 2.5-cm subepithelial mass originating from the muscularis propria, without lymph node involvement (> Fig.1b). A diagnosis of a gastrointestinal stromal tumor (GIST) was made by fine-needle biopsy, and the patient was referred for endoscopic full-thickness resection (EFTR) of the mass.

Initially a semicircumferential incision was made with an IT knife (Olympus, Tokyo, Japan) (\Fig.2a; \Video 1), then the lesion was lifted upward and well exposed using clip-with-line traction
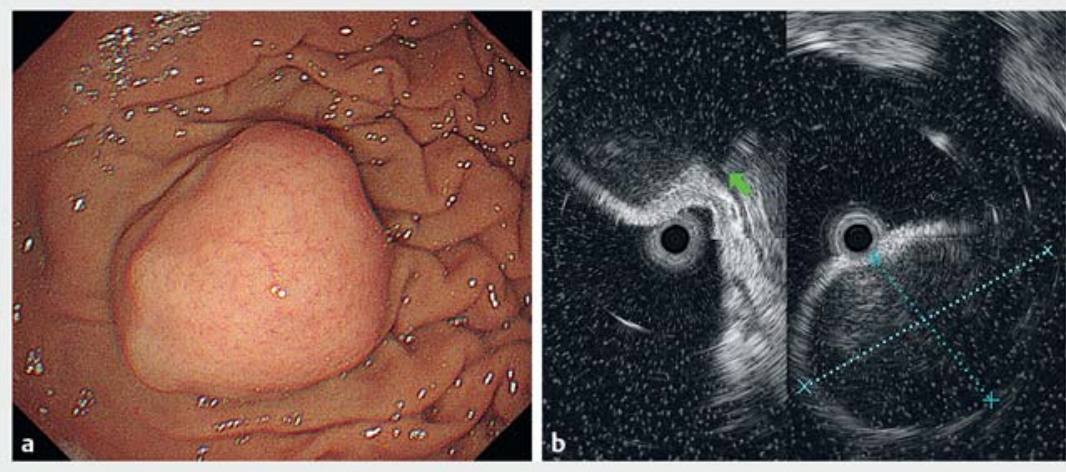

Fig. 1 A huge gastrointestinal stromal tumor (GIST) seen on: a endoscopic view showing a $2.5-\mathrm{cm}$ submucosal tumor in the fundus of the stomach; $\mathbf{b}$ endoscopic ultrasonography view showing the GIST was originating from the muscularis propria layer.
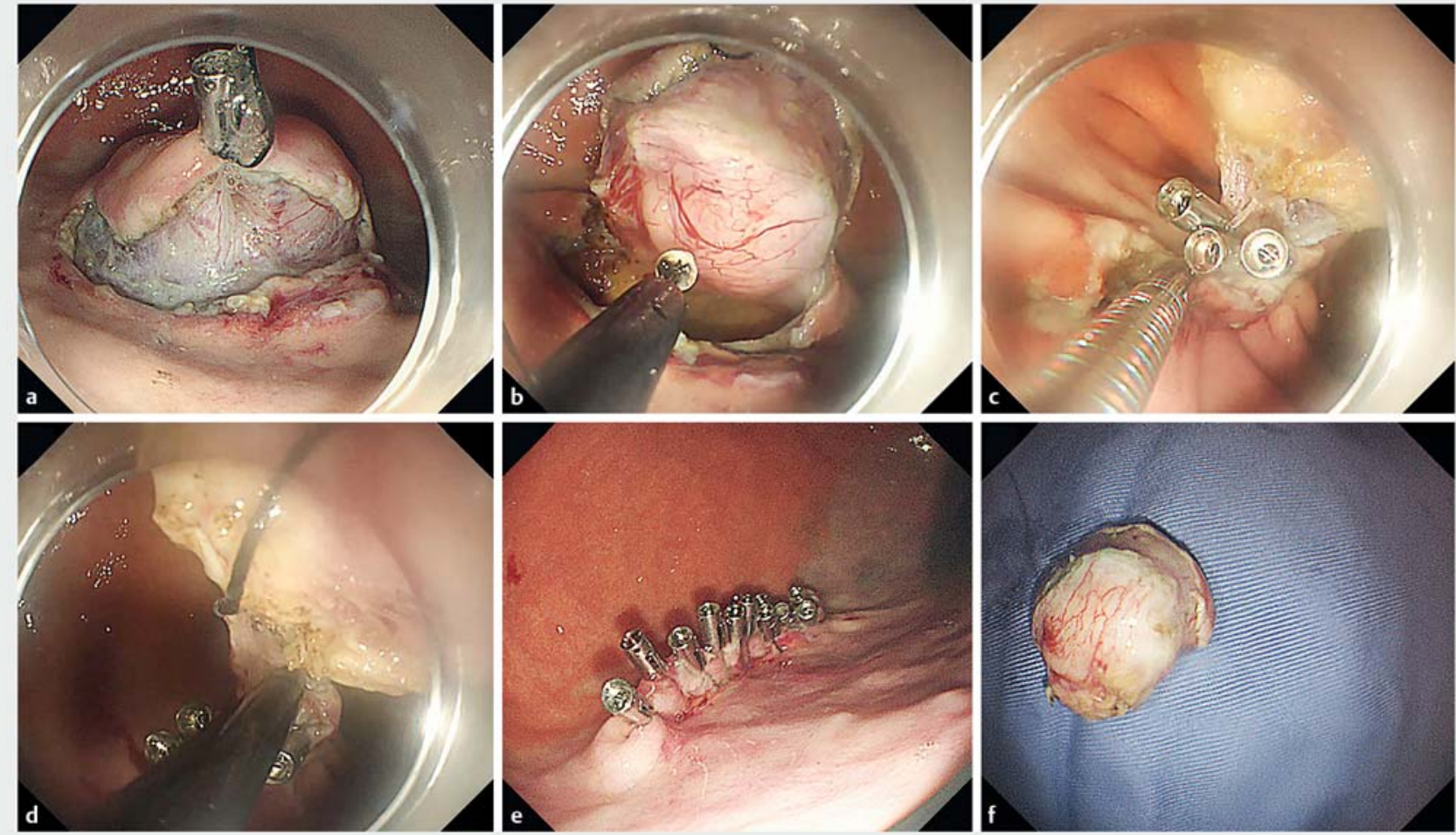

- Fig. 2 Stages in the endoscopic full-thickness resection of a gastric fundal gastrointestinal stromal tumor (GIST) using the preclosure technique and traction method: a the huge GIST was revealed after peritumor gastric mucosa and submucosa dissection; $\mathbf{b}$ full-thickness resection was performed around the tumor using clip-with-line traction; $\mathbf{c}$ once most of the tumor had been excavated, the gastric perforation was preclosed using clips with the help of the clip-with-line traction; $\mathbf{d}$ the remaining tissues adherent to the tumor were dissected; e the gastric defect was completely closed with a line of clips. $\mathbf{f}$ Macroscopic appearance of the resected specimen showing the full-thickness structure of the peritumor gastric wall. 


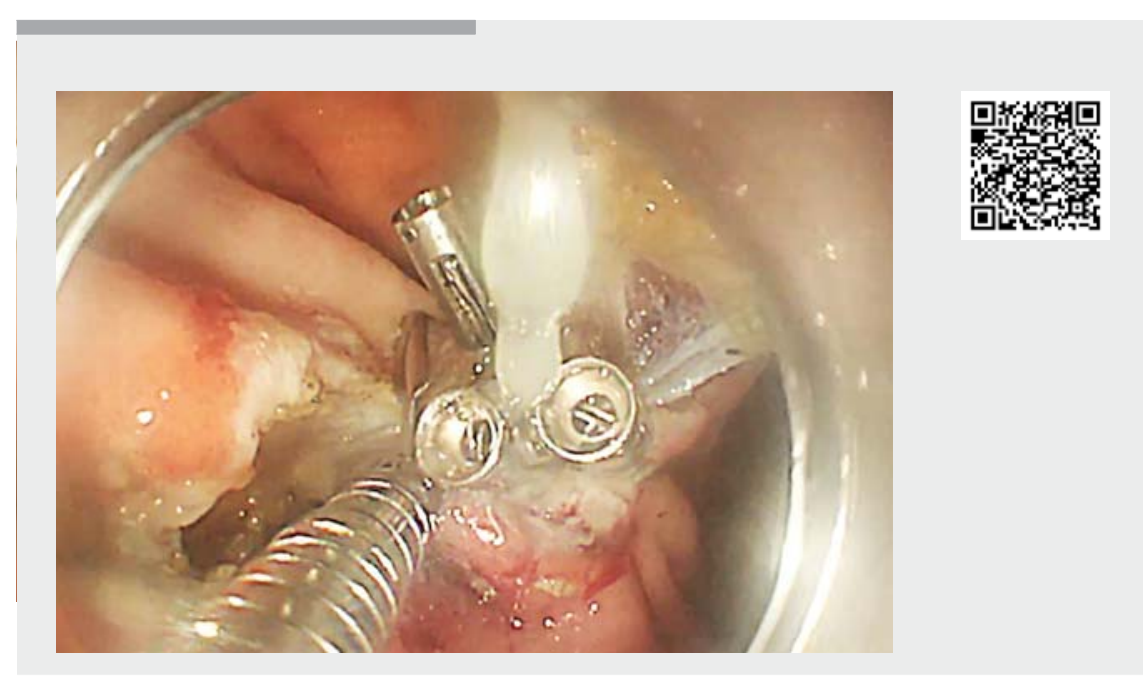

$\checkmark$ Video 1 Preclosure technique and traction method facilitating endoscopic full-thickness resection (EFTR) of a gastrointestinal stromal tumor (GIST) in the gastric fundus. The lesion was lifted with normal saline solution from the anal side. With adequate expansion of the submucosal space, the semicircumferential incision of the mucosa was performed with an IT knife. After the lesion had been clearly identified, the clip-with-line traction was applied to adequately expose the mass. As the mass was well lifted, the submucosal space beneath the lesion was quickly dissected under a constant tension. Because the GIST was tightly adherent to the underlying muscularis propria, EFTR was carried out until most of it had been freed from the gastric wall. The gastric wall defect was then preclosed with clips in a "side to center" manner by linearly lifting the clip-with-line traction. As the mass was effectively prevented from falling into the peritoneal cavity, it was completely resected and safely extracted. The remaining defect was fully closed with additional clips.

( Fig. 2 b). Most of the GIST was rapidly dissected off the muscularis propria, leaving approximately a 2.6-cm defect. Owing to worries over the mass falling into the abdominal cavity and being unable to close the defect, the defect was preclosed linearly with clips under clip-with-line traction before removal of the mass ( Fig. $\mathbf{2 c}$ ). The GIST was then safely and entirely resected ( $>$ Fig. 2 d) and reliable closure of the defect was achieved with additional clips ( $>$ Fig. 2 e). The extracted specimen displayed complete resection with adherent full-thickness gastric tissue ( $\triangleright$ Fig. $2 \mathbf{f}$ ). The patient had no adverse events and was discharged after 3 days.

Although various methods and devices for gastrointestinal wall closure have sprung up in recent years, most of them require complex or specialized equipment and are technically challenging [1 4]. In this study, we demonstrated that
The authors

Xiao-gang Liu *, Zi-yang Chen *, Yun-chao Yang, Ren-yi Zhang, Wei-hui Liu Department of Gastroenterology and Hepatology, Sichuan Academy of Medical Sciences \& Sichuan Provincial People's Hospital and School of Medicine, University of Electronic Science and Technology of China, Sichuan Province, China

Corresponding author

\section{Wei-hui Liu, MD, PhD}

Department of Gastroenterology and Hepatology, Sichuan Academy of Medical Sciences \& Sichuan Provincial People's Hospital, Chengdu, Sichuan Province, 610072, China audiliu12@163.com

\section{References}

[1] Azzolini F, Cecinato P, lori V et al. Endoscopic full-thickness resection for suspected residual rectal neuroendocrine tumor and closure of the defect with a new suturing system. Endoscopy 2015; 47 (Suppl. 01): E556E557

[2] Aslanian HR, Sethi A. ASGE Technology Committee. et al. ASGE guideline for endoscopic full-thickness resection and submucosal tunnel endoscopic resection. VideoGIE 2019; 4: 343-350

[3] Chiu PW, Phee S], Wang Z et al. Feasibility of full-thickness gastric resection using master and slave transluminal endoscopic robot and closure by Overstitch: a preclinical study. Surg Endosc 2014; 28: 319-324

[4] Zhang Y, Wang X, Xiong G et al. Complete defect closure of gastric submucosal tumors with purse-string sutures. Surg Endosc 2014; 28: 1844-1851

\section{Bibliography}

\section{Endoscopy_UCTN_Code_TTT_1AO_2AG}

\section{Competing interests}

The authors declare that they have no conflict of interest.
DOI https://doi.org/10.1055/a-1109-2445

Published online: 17.2.2020

Endoscopy 2020; 52: E293-E294

(C) Georg Thieme Verlag KG

Stuttgart $\cdot$ New York

ISSN 0013-726X

\footnotetext{
* Contributed equally to this work
} 